\title{
Models for pheromone evaluation in Ant Systems for Mobile Ad-hoc networks
}

\author{
Fernando Correia \\ Inesc-ID and Instituto Superior Técnico, Lisboa, Portugal \\ Victor J. Lobo \\ Portuguese Naval Academy and ISEGI-UNL, Lisboa, Portugal \\ Teresa Vazão \\ Inesc-ID and Instituto Superior Técnico, Lisboa, Portugal
}

\begin{abstract}
On a mobile ad-hoc network environment where the resources are scarce, the knowledge about network's link state is essential to optimize the routing procedures. This paper presents a study about different pheromone evaluation models and how they react to possible changes in traffic rate. Observing how the pheromone value on a link changes, it could be possible to identify certain patterns which can indicate the path status.

For this study, it was compared the behavior of the Ant System evaluation model, with a Temporal Active Pheromone model (a biological approach) and a Progressive Pheromone Reduction model with / without maximum pheromone limit.
\end{abstract}

Key words: Biological Routing, Swarm intelligence, Ant system

\section{Introduction}

The Ant System (AS) algorithm, originally proposed by Dorigo et. al [1], is a cooperative search algorithm based on social ant's behavior. The ants are able to find good path solutions, between the nest (source) and the food (destination). The best routes discovered allow to attract more ants, and the ant's community will converge to one best solution.

In this field, it has been presented several algorithms which modulate ant's behavior, in its food search procedure $[2,3]$. In Dorigo et.al "Distributed Optimization by Ant Colonies" [4], it is presented

Email addresses: fcorreia@tagus.inesc-id.pt (Fernando Correia), vlobo@isegi.unl.pt (Victor J. Lobo), teresa.vazao@tagus.ist.utl.pt (Teresa Vazão). a new group of routing models, named as Swarm Intelligence-based Routing [5].

When observing the ants, they have communication procedures, which result on path convergence to the shortest route ever found. In this procedure, the environment variations that enclose the ants, are reflected in the path choice.

As long as the ants are traveling through the path, a chemical substance named as pheromone is left. The pheromone allows the ants to return to the nest, but also, to attract more ants to that path. This is a communication mechanism between the ants. This way to communicate called stigmergy [6], is an indirect form of communication used by ants. It can present the environment changes and how the ants could adapt itself to the new reality. 
This communication mechanism also can be applied to fixed or mobile data networks. Instead of use ants, it is possible to use data or control packets (virtual ants), which can modulate ant's behavior when they travel through the network. Like in the real environment, the traffic flow variations will be reflected in the pheromone value present in the links between the nodes. The change of pheromone value can indicate the state of the network.

The use of these procedures in Mobile Ad-hoc Networks (MANET) can be very useful. The ad-hoc networks has scarce bandwidth and a constant change of topology. the identification of bottle-necks (link congestion) or broken links (communications failure between nodes) through the analysis of pheromone level variation, can help to create a picture of the network state. The identification of this problems, allows the routing procedures to choose the best paths to forward the traffic and perform an optimization of the network resources.

This paper presents a study about pheromone level variation, applied to the MANET's, when in presence of different levels of load in the network. Analyzing the pheromone behavior, it is possible to gather information about the network state without the use of extra control packets. Due this, it is possible to detect on an earlier stage, problems in the network and re-direct the traffic to alternative routes. This action can minimize the loss of data packets and increase the network performance.

For this study, it was used the Simple Ant Routing Algorithm (SARA) [7] developed by the author, for Mobile Ad-hoc Networks. To evaluate the pheromone intensity, it was considered four different models of pheromone reinforce / evaporation and observed the time response of each one to different traffic situations.

The remain of the document is organized as follows: in section 2 is presented related work about the pheromone evaluation; section 3 describes the concept used to define the models to be tested and presents the simulation results; finally, section 4 presents the conclusions reached and the planned future work.

\section{Related work}

When the ants start to explore the environment near the nest to search the food, they present a capability to find and maintained the best (short) path. This path will be the one with higher value of pheromones, and so, it will attract more ants.

To model ant's behavior on mobile networks, it should be used routing agents and data packets to act like virtual ants. This virtual ants will leave a pheromone trail in the network, to signal the routes between the source node and destination node. The routes with a higher value of pheromones, should indicate the best routes in the network.

The mechanism named as stigmergy, use the pheromones as an indirect form of communication. This can be done, because the pheromone has a life time period, in which it is active. The intensity observed on the path, is related with capacity of the ants to use it. The path with a higher pheromone value will attract more ants and a path convergence is observed.

However, in mobile network, the convergence of virtual ants to one best route, could not be a desirable situation. In mobile networks, the bandwidth is scarce and an overload of traffic can cause congestion problems. Because of this, the routing procedures must have information based not only the pheromone value, but also how this value is evaluated. This decisions will influence the reinforce / evaporation of the pheromones. Another problems that actually it is necessary to solve, is an early best route identification. This problem will restrict the search for paths in the network.

In the AS model, the procedures to choose the route to send the packets to the next hop is probabilistic. It uses an heuristic based on pheromone value present on a link. The eq. (1) presents the pheromone evaluation.

$$
\begin{aligned}
& \tau_{(i, j, t)}=\rho \tau_{(i, j, t-1)}+\sum_{k=1}^{n} \Delta \tau_{(i, j, t)}^{k} \\
& \Delta \tau_{(i, j, t)}^{k}= \begin{cases}\frac{1}{d_{i, j}}, \text { if }(i, j) \in \text { path use by ant } k \\
0\end{cases}
\end{aligned}
$$

where $\tau_{(i, j, t)}$ is the pheromone level in the link between node $i$ and node $j$ at time t. $\rho(0<\rho<$ 1 ) indicates the pheromone evaporation value and $\Delta \tau_{(i, j, t)}^{k}$ is the pheromone reinforce value, calculated upon the distance $d_{(i, j)}$ traveled by the ant $k$ from node $i$ to node $j . n$ is the number of ants used to find the path.

When an ant reaches a point where it has several routes to choose, it is necessary to decide where to go. In data networks, the AS model can forward the data traffic to the next hop, on an hop-by-hop basis. 
It uses a probabilistic procedure to decide where to send the packets, in accordance with the metrics associated to the model. In AS, the metric is based on the pheromone value assign to each link between two nodes in the network.

The pheromone intensity is implicit related with the distance traveled by the virtual ants, it means, the shorter paths has a higher refresh rate than the other ones.

When the procedure used to choose the next hop is probabilistic, the better path found for a certain session could not be indeed the best path in the network. This an earlier path convergence problem, which has been studied by several researchers. To solve this problem, it has been studied several models which try to reduce the early convergence effect. Models like the MAN-MIN Ant System (MMAS) [8,9], the Minimum Pheromone Threshold Strategy (MPTS) [10], the Elitist Strategy for Ant System (ASe) [11], the Rank-based version of Ant System (ASrank) [12], or the Pheromone Trail Centralization (PTC) [13].

The above models can be applied in the search procedure to find the short path, considering the traffic dynamics in fixed networks, in which the network topology doesn't change. With this technics, it is possible to identify the short paths, related with the distance traveled and the propagation time in the network.

Most of the studies about AS, has been focus to solve static problems, like the Travel Sales Man. The optimization problems that it is possible to find in the real world, are not statics, and so, it is necessary to observe the dynamic component.

The traffic congestion as describe by Eyckelhof and Snock et.al, in Ant System algorithm to applied to Dynamic Travel Salesman Problem (ASDTSP) [14], driven by bottlenecks in car roads, is a problem that can be observed on a daily basis. The traffic density is dynamic by nature and find an optimum solution to regulate the traffic is the challenge. In this area of interest, models like Dynamic System for Avoiding Traffic Jam (DSATJ) [15] are proposed.

The procedures to route data packets in a network, shows a similar behavior like the procedures that are used to control the car flow on the roads. It is possible to gather important information about the state of the road / network, taken in consideration the occupation density of the road / communication links, though the variation of the pheromone level, which was left by cars / virtual ants (data and control packets).

The application of AS algorithm to mobile networks, can integrate the network state information, with low overhead (stigmergy). However, due the frequent change of topology, the use of pheromones must be adapted to this new reality.

The pheromones can be a network state indicator. The traffic flow variation, which could be a direct result of a bottleneck or broken link, is reflected in the pheromone level through the time. This fact can help the routing procedures to balance the load through other paths or start to search for new routes.

\section{Pheromone variation models}

In the model proposed by Dorigo for Ant System [1], the evaporation rate is related with a parameter $\rho$ and the quantity of pheromones deposit on the track. The pheromones are dependent by the number of ants that just passed through the link and the distance traveled to the destiny.

This model is used to identify the best paths on a wired network. With this, it is possible to create a heuristic, which can gather information about the network state - best short routes to destiny.

The Ant System model works with the distance that the ants must travel through the network, but on mobile ad-hoc networks, where the topology is on a constant change, there are other factors which are also important. In MANET's, the best path must have a dynamic solution, in accordance with the nodes mobility.

The state of a path, can be evaluated by the variation of the pheromone value. When a path has available resources to transport the packets, the pheromone refresh rate will be the same as long as the transfer session is active. So the pheromone value will not present considerable changes. However, if there is a bottleneck in the path, the refresh rate will change and this situation could be observe in the pheromone value.

In this study, we intend to identify possible problems in the network, like congestion, broken links, etc, on an earlier stage and help the route procedures to choose the best routes available. To reach this aim, we are going to use four pheromone models of reinforcement/evaporation and evaluate them on a optimum network situation.

The simulations were executed using the NS2 simulator, version 2.31. For each one of the tests proposed, 30 seconds simulation runs were executed. 
The $802.11 \mathrm{~b}$ protocol was used for the MAC layer and the radios used the Two Ray Ground Propagation model and had a receiving range of 100 meters. The traffic was generated by one session, with $50 \mathrm{kbps}$ rate and the type was CBR.

The pheromone evaluation models used were:

- Ant System pheromone model.

- Temporal active pheromone model.

- Progressive pheromone reduction model.

- Progressive pheromone reduction with maximum value model.

\subsection{Ant System pheromone model}

In the AS model, the evaluation of the pheromone level is presented in eq. (1). It is defined a $\rho$ value $(0<\rho<1)$ which represents the evaporation rate and $\Delta \tau_{(i, j, t)}^{k}$ value which is the reinforcement of the pheromones, based on the number of ants that passed on the path. The $\rho$ value must be related with the data transfer rate and the sample interval applied to calculate the pheromone value.

The aim of this study is to observe the traffic behavior through the pheromone level variation, so the eq. (1) was change to:

$\tau_{(i, j, t)}=\rho \tau_{(i, j, t-1)}+\sum_{k=1}^{n} \Delta \tau_{(i, j, t)}^{k} \wedge \Delta \tau_{(i, j, t)}^{k}=1$

In the model under test define by eq. (2), we want the pheromone level be dependent of the network state. The distance traveled by the virtual ants will be implicit in the pheromone intensity present in the path.

This is a multiplicative low complexity model. It must know the number of ants that just pass through the link, during the sample time and the pheromone value in the previous sample time $(t-1)$.

To simulate this model, it was considered three different values of $\rho$. The time used between samples was 1 second. The fig. 1 presents the pheromone variation.

With this model, it is possible to identify three different phases:

(i) learning phase: the reinforcement of the pheromone value is related with the $\rho$ value and the traffic rate which flows through the path. During this phase the pheromones will increase on a logarithmic way, until reach a stable value.

(ii) maintenance phase: the pheromone intensity will be the same with little changes, if the link

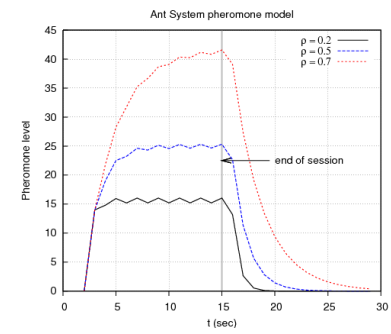

Fig. 1. Pheromone behavior on AS model

maintains the capacity to transfer the traffic, i.e the reinforcement rate equalize the evaporation rate.

(iii) evaporation phase: when the traffic stops flowing through the path, the pheromone level decreases rapidly and then slows down until reach ' 0 '. This phase could also be noted if there is a problem in the path, due traffic jam or a broken link.

The Ant System pheromone model can reach a stable value when the link capacity has enough resources to transport the packets. It presents a fast grow rate until reach a stable value. The intensity of the pheromones depends the evaporation value of $\rho$.

When the data transfer session ends, or when in a presence of a bottleneck due link problems, the AS model will react to this and present a fast decrease of the pheromones.

This reaction could be useful when applied to systems which needs a fast response. However, on a network, changes on traffic transfer rate like packet jitter or burst of packets, could create a response similar to link bottleneck and give an wrong information about the network state.

The response to the pheromone variation should have a smaller decrease slope rate, which will accommodate the traffic rate variations.

\subsection{Temporal active pheromone (TAP) model}

The Temporal active pheromones model, tries to modulate a real behavior of the pheromones. When a real ant deposits a pheromone in the trail, the pheromone is active for a certain period of time.

The pheromone activity presents a decrease curve of intensity, which is very complex to represent. To perform a good approach of this model, it is considered that when the pheromone activity decrease, it will have a value equals to ' 0 '. The eq. (3) shows the pheromone activity. 


$$
\begin{aligned}
\tau_{t} & =\left\{\begin{array}{lr}
1(\text { active }) & t_{\text {start }}<t<t_{\text {start }}+\delta \\
0(\text { evaporation }) & t>t_{\text {start }}+\delta
\end{array}\right. \\
T_{t} & =\sum \tau_{t}
\end{aligned}
$$

$\tau_{t}$ represents the state of a single pheromone at time $t$ and $\delta$ is the activity period. A pheromone is active in the interval $\left[t_{\text {start }}, t_{\text {tart }}+\delta\right]$. The pheromone intensity on a link $\left(T_{t}\right)$ is the sum of all active pheromones deposited on that link.

To modulate the pheromone behavior with a timelife parameter, has the advantage of create an evaluation model which looks like the real pheromones. However, to simulate this model on each node, it is required more resources on each network node. The node must have enough memory to keep update tables with pheromone and computational capacity to deal with the timers responsible to define the pheromone activity.

The timers must be set for a value which can give a real approach of the pheromone activity. The fig. 2 represents this model's simulation. For this simulation, the pheromone active was set to $500 \mathrm{~ms}$, $1 \mathrm{sec}$ and $2 \mathrm{sec}$. The timer is set to be active every $50 \mathrm{~ms}$.

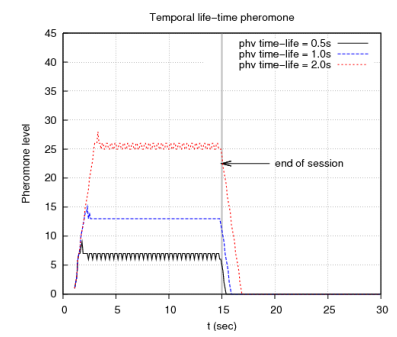

Fig. 2. Pheromone behavior on Temporal active model

In fig. 2, like in AS model, it can be possible observed three different phases during pheromone evaluation analysis of this model.

The learning phase is related with the time-life of the pheromones and transfer rate through a link. The pheromone intensity will grow as long as the pheromones are in the active period. The growing rate is the same during this phase, while there isn't any problem in the network.

The maintenance phase starts after a period of time equals to the time defined for the pheromone activity. The pheromone intensity will maintain the same value, as long as the refresh rate is the same as the evaporation rate. In this phase, the network status can be translated through the capacity of a link to maintain the pheromone value bounded, while the transfer session is active. Any problems in the net- work, could be observed in the pheromone intensity variation, during this phase.

When the data transfer session stops, the reinforcement rate will decrease to '0'. The evaporation phase, is the period required for the pheromones to cease their activity, and is equal to the pheromone time-life.

With this evaluation model, the network state can be observe in the pheromone level variation during the maintenance phase. When the network has resources to transfer the packets without losses or excess delay, the pheromone level variation will be almost ' 0 '. However, when in presence congestion or broken links, the pheromone refresh rate will change and the pheromone intensity variation could be an indicator.

The pheromone activity decrease rate is also related with jitter or burst of data packets situations and will affect the pheromone value. However, because the decrease rate is linear, these effects can be absorbed and the link can regain its pheromone value when disappear.

The pheromone time-live model is more stable to the network state changes than the Ant System pheromone model. The stability parameter is assign to the pheromone time-life, which will act as a buffer to the pheromone refresh rate. This model will be slower to detect network problems than the AS model.

\subsection{Progressive pheromone reduction (PPR) model}

The Progressive Pheromone Reduction is a model which uses lower node resources to compute the pheromone intensity.

The pheromone value present on a link, increases as long as the traffic flows through it. The increase rate is related with the link's capacity to transport the packets. Variations in the traffic, can be observed in the pheromone increase rate.

The refresh and evaporation procedures, are defined in eq. (4).

\section{Refresh :}

$\tau_{t}=\tau_{t-1}+\rho$

\section{Evaporation :}

$$
\begin{aligned}
& \tau_{t}=\tau_{t-1}-\alpha \\
& \alpha=\left\{\begin{array}{l}
1 \quad \text { with link activity } \\
\alpha * 2 \text { without link activity }
\end{array}\right.
\end{aligned}
$$


Where $\tau_{t}$ is the actual pheromone value and $\rho$ represents the increase value when a packet go through the link. On a constant transfer rate environment, the pheromone value will increase on the same rhythm. When this rhythm changes, it could means that there are problems in the network. The analysis of the first derivate of the pheromone variation, can quantify the problem with a certain degree of probability. For this model, it was used $\rho=1$.

The pheromone evaporation procedure is executed periodically. Every time this procedure is called, the pheromone value is decreased by $\alpha$. If the packets are flowing through the link, $\alpha=1$, however, if the traffic ceases, $\alpha$ will duplicate the value every time the evaporation procedure is called and there is no activity in that link. This way, small traffic anomalies caused by jitter, packet burst or route repair procedures, will not affect the pheromone value, but when in the presence of a broken link, this will cause a progressive pheromone evaporation and without reinforcement the pheromone value falls rapidly to ' 0 '.

The fig. 3 shows the behavior of this model.

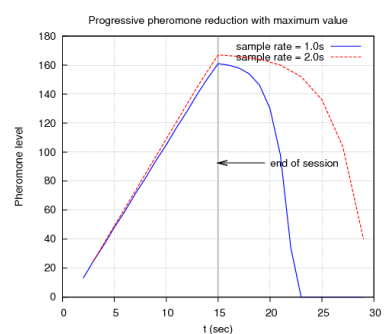

Fig. 3. Progressive pheromone reduction model

This model presents two valid phases: the maintenence phase and the evaporation phase.

The maintenance phase is observed as long as the traffic flows through the link. The evaporation phase is progressive and happens when the traffic ceases its activity.

The Progressive Pheromone Reduction is a simple model to evaluate the pheromone value, which attends the variations of pheromone intensity to identify and classify the network state, with a certain level of probability.

The evaporation procedure, allows that small variations in the traffic rate, doesn't be notice in the pheromone global value and the path will continue to be marked as valid. When the traffic stops in the network, the pheromone activity also stops and the route can be released.

\subsection{Progressive pheromone reduction with maximum value model}

The Progressive Pheromone Reduction with Maximum Value (PPR-MV) model, is similar to the model presented in sec. 3.3, with a slight difference: the pheromones present in a link are limited to a maximum value per traffic session.

In this model, the pheromones have two levels of evaluation. One level is associated to a data session and the other level to a link between nodes. The link state indicator, is represented by the variation of the sum of all pheromones assign to the sessions present on that link. If a link has enough resources to accommodate new data session, then the sum of pheromones should increase on a direct proportion of number of sessions.

On an ad-hoc network, where all the nodes have mobility, it is very important to know the amount available resources to choose the next hop in the path. Limiting the pheromone to a maximum value, the variations on the pheromone intensity would not be enough to identify jitter, packet burst or congestion situations, but when the traffic ceases, the pheromone intensity will progressive returns to ' 0 '.

The eq. (5) represents the PPR-MV.

Refresh :

$\tau_{t}= \begin{cases}\tau_{t-1}+\rho & \text {;if } \tau_{t-1}<M A X_{-} V A L U E \\ M A X_{-} V A L U E & \text {;if } \tau_{t-1}=M A X_{-} V A L U E\end{cases}$

Evaporation :

$\tau_{t}=\tau_{t-1}-\alpha$
$\alpha=\left\{\begin{array}{l}1 \quad \text {;with link activity } \\ \alpha * 2 \quad \text {;without link activity }\end{array}\right.$

The behavior of PPR-MV is shown in fig. 4 .

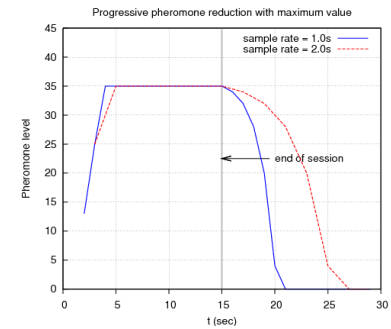

Fig. 4. Progressive pheromone model with maximum value

This model has three phase in the pheromone evaluation. The learning phase is related with the ses- 
sion packet rate in the path and it end when the pheromone value reach the maximum that has been selected. While the data transfer session is active, the maximum pheromone value is maintained.

Like in PPR model, small traffic rate variations are absorb by the progressive evaporation procedure and the pheromone value shouldn't be almost the same. When the data transfer session ends, the pheromone progressive decreases to ' 0 '.

With PPR-MV, it could be difficulty to identify the network state, due slow reaction to the network state changes. It has the ability to distinguish the active routes, from those which aren't in use.

\section{Conclusions}

This paper presents a study with different pheromone evaluation models. Each of those models have different characteristics and could be used to indentify the network state, with a certain degree of probability.

It was used four evaluation models: the Ant System model, the Temporal active pheromone model, the Progressive pheromone reduction model and the Progressive pheromone reduction with maximum value model.

The pheromone variation could be an indicator of the network state, without introducing overhead. Modeling the pheromone behavior, through this four models, it could be possible to create heuristics to be used by the routing procedures. This way, it is possible to optimize the network resources.

Due the characteristics of each model, they can be use in specific scenarios, where a fast response to traffic situations can be balanced with a stable pheromone activity.

For future work, it is intended that an evaluation about the behavior of the pheromone models, on network situations like link congestion and broken links, and correlate these studies with the possibility to create a virtual image of the network state.

\section{References}

[1] M. Dorigo, V. Maniezzo, and A. Colorni. Ant system: optimization by a colony of cooperating agents. Systems, Man, and Cybernetics, Part B, IEEE Transactions on, 26(1):29-41, Feb 1996.

[2] Zhenyu Liu, Marta Z. Kwiatkowska, and Costas C. Constantinou. A biologically inspired QoS routing algorithm for mobile ad hoc networks. In $A I N A$, pages 426-431, 2005.
[3] Reza GhasemAghaei; Md. Abdur Rahman; Wail Gueaieb; Abdulmotaleb El Saddik. Ant ColonyBased Reinforcement Learning Algorithm for Routing in Wireless Sensor Networks. In Instrumentation and Measurement Technology Conference Proceedings, pages 1-6. IEEE, 1-3 May 2007.

[4] A. Colorni, Marco Dorigo, and Vittorio Maniezzo. Distributed optimization by ant colonies. In F. J. Varela and P. Bourgine, editors, Towards a Practice of Autonomous Systems: Proceedings of the First European Conference on Artificial Life, pages 134-142. MIT Press, Cambridge, MA, 1992.

[5] F.; Gambardella L.M. Di Caro, G.; Ducatelle. Swarm intelligence for routing in mobile ad hoc networks. In Swarm Intelligence Symposium, pages 76-83. SIS 2005, 8-10 June 2005.

[6] Guy Theraulaz and Eric Bonabeau. A brief history of stigmergy. Artificial Life, 5(2):97-116, 1999.

[7] F. Correia and T. Vazao. Simple ant routing algorithm. Information Networking, 2008. ICOIN 2008. International Conference on, pages 1-8, Jan. 2008.

[8] T. Stutzle and H. Hoos. Max-min ant system and local search for the traveling salesman problem. Evolutionary Computation, 1997., IEEE International Conference on, pages 309-314, Apr 1997.

[9] Thomas Stützle and Holger H. Hoos. MAX-MIN ant system. Future Generation Comp. Syst, 16(8):889-914, 2000.

[10] Phen Chiak See, Kuan Yew Wong, and Komarudin. A technique for improving the max-min ant system algorithm. Computer and Communication Engineering, 2008. ICCCE 2008. International Conference on, pages 863-866, May 2008.

[11] Marco Dorigo. Optimization, Learning and Natural Algorithms. PhD thesis, Politecnico di Milano, Italy, 1992.

[12] Bernd Bullnheimer, Richard F. Hartl, and Christine Strauss. An improved ant system algorithm for the vehicle routing problem, December 081997.

[13] Song Zheng, Guangxing Zhang, and Zekui Zhou. Ant colony optimization based on pheromone trail centralization. Intelligent Control and Automation, 2006. WCICA 2006. The Sixth World Congress on, 1:3349-3352, 2006.

[14] Casper Joost Eyckelhof and Marko Snoek. Ant systems for a dynamic TSP. In Marco Dorigo, Gianni Di Caro, and Michael Sampels, editors, Ant Algorithms, volume 2463 of Lecture Notes in Computer Science, pages 8899. Springer, 2002.

[15] P. Bedi, N. Mediratta, S. Dhand, R. Sharma, and A. Singhal. Avoiding traffic jam using ant colony optimization - a novel approach. Conference on Computational Intelligence and Multimedia Applications, 200\%. International Conference on, 1:6167, Dec. 2007. 